\title{
openheart Efficacy and safety of non-vitamin K antagonist oral anticoagulants compared with warfarin in patients with atrial fibrillation
}

\author{
Leif Friberg, ${ }^{1}$ Jonas Oldgren ${ }^{2}$
}

To cite: Friberg L, Oldgren J. Efficacy and safety of non-vitamin $\mathrm{K}$ antagonist oral anticoagulants compared with warfarin in patients with atrial fibrillation. Open Heart 2017;4:e000682. doi:10.1136/ openhrt-2017-000682

- Prepublication history and additional material for this paper are available online. To view please visit the journal (http:// dx.doi.org/10.1136/openhrt2017-000682)

Received 28 June 2017 Revised 27 July 2017 Accepted 22 August 2017

\section{(a) CrossMark}

${ }^{1}$ Department of Clinical Sciences at Danderyd Hospital, Karolinska Institute, Stockholm, Sweden

${ }^{2}$ Department of Medical Sciences, Cardiology and Uppsala Clinical Research Center, Uppsala University, Uppsala, Sweden

Correspondence to Professor Jonas Oldgren; jonas. oldgren@ucr.uu.se

\section{ABSTRACT}

Aims Non-vitamin $\mathrm{K}$ antagonist oral anticoagulants (NOACs) were in pivotal randomised controlled trials at least non-inferior to warfarin for stroke prevention in atrial fibrillation, but time in therapeutic range (TTR) for warfarin was lower (mean 55\%-65\%) than in Swedish general care where TTR is $>70 \%$. We compared efficacy and safety of NOACs and warfarin treatment for stroke prevention in Sweden.

Methods Retrospective cohort study of all non-selected oral anticoagulation naïve atrial fibrillation patients with first prescription for NOACs or warfarin between December 2011 and December 2014, excluding patients with mitral stenosis or mechanical valvular prosthesis. Data were obtained from cross-linked national registers, propensity scores were used as continuous covariates, and associations between treatment and outcomes were evaluated by multivariable Cox regressions.

Results The study comprised 18638 patients on NOAC and 49418 on warfarin treatment, with 90204 patientyears follow-up. Age (mean) was 73.4 vs 73.7 years, $\mathrm{p}<0.001$, and $\mathrm{CHA}_{2} \mathrm{DS}_{2}-\mathrm{VASc}$ points (mean) 3.38 vs 3.24 , $\mathrm{p}<0.001$, in NOAC and warfarin groups, respectively. HRs $(95 \% \mathrm{Cl})$ for NOACs versus warfarin were $1.04(0.91-1.19)$ for all-cause stroke or systemic embolism, 1.16 (1.001.35) for ischaemic stroke, $0.85(0.76-0.96)$ for major bleeding, $1.22(1.01-1.46)$ for gastrointestinal bleeding, $0.60(0.47-0.76)$ for intracranial haemorrhage and 0.89 (0.81-0.96) for all-cause mortality.

Conclusion In this large non-selected anticoagulation naïve Swedish atrial fibrillation cohort, the risks for allcause stroke or systemic embolism were similar with NOACs and warfarin, but NOACs were associated with significantly lower risks of all-cause mortality, major bleeding and intracranial haemorrhage but higher risk of gastrointestinal bleeding. Better safety suggests NOACs as preferred treatment for patients with atrial fibrillation starting oral anticoagulation.

\section{INTRODUCTION}

Warfarin and other vitamin $\mathrm{K}$ antagonists (VKA) have for long time been established as a highly effective stroke prevention treatment in atrial fibrillation. In a meta-analysis of in total 2900 patients based on six trials conducted more than two decades ago,

\section{KEY QUESTIONS}

What is already known about this subject?

- Non-vitamin $\mathrm{K}$ antagonist oral anticoagulants (NOACs) were in pivotal randomised controlled trials non-inferior to warfarin for stroke prevention in atrial fibrillation (AF), but the mean time in therapeutic range (TTR) of $55 \%-65 \%$ for warfarin in these studies was substantially lower than the $\geq 70 \%$ recommended in the European AF guidelines

- In Sweden, patients with AF receiving warfarin treatment in clinical trials as well as non-selected AF patients in general practice or at anticoagulation clinics have repeatedly been shown to have mean TTR above the recommended $70 \%$.

What does this study add?

- In this large non-selected anticoagulation naïve Swedish AF cohort, the risks for all-cause stroke or systemic embolism were similar with warfarin and NOACs (apixaban, dabigatran and rivaroxaban), but NOACs were associated with significantly lower risks of all-cause mortality, major bleeding and intracranial haemorrhage than warfarin.

How might this impact on clinical practice?

- NOACs should be the preferred treatment for patients with AF starting oral anticoagulation for stroke prevention even in healthcare settings where warfarin treatment is of high quality.

warfarin treatment was shown to reduce stroke by approximately two-thirds and total mortality by a quarter, but VKA have also been associated with elevated bleeding risk compared with placebo or no treatment. ${ }^{12}$ Due to a narrow therapeutic range, the benefit of warfarin is highly dependent on time within the therapeutic range (TTR) measured as international normalised ratio (INR) 2.0-3.0..$^{3-5}$

Non-vitamin $\mathrm{K}$ antagonist oral anticoagulants (NOAC) were approved for stroke prevention in patients with atrial fibrillation in 2010. All currently available NOACs, 
apixaban, dabigatran, edoxaban and rivaroxaban, have been shown to be at least non-inferior, dabigatran $150 \mathrm{mg}$ and apixaban even superior, for stroke prevention compared with dose-adjusted warfarin in randomised controlled trials (RCT), comprising $>71000$ patients in total. ${ }^{6-9} \mathrm{~A}$ meta-analysis of these trials ${ }^{10}$ showed a $13 \%$ reduction in all-cause stroke or systemic embolism and a $20 \%$ reduction in major bleeding events. In these trials, mean TTR was $55 \%-65 \%$, with large variations between countries, centres and patients. Thus, the vast majority of warfarin-treated study patients had TTR lower than the desired minimum of $70 \% .{ }^{11}{ }^{12}$ Observational data from non-selected patients in usual clinical practice worldwide often exhibit even lower TTR. ${ }^{13-15}$ In contrast, results from Swedish patients enrolled in clinical trials ${ }^{16}$ as well non-selected patients in general practice or at anticoagulation clinics ${ }^{1819}$ have repeatedly shown mean TTR above the recommended $70 \%$.

We aimed to evaluate the efficacy and safety of NOACs compared with high standard warfarin management in non-selected patients with atrial fibrillation not previously exposed to oral anticoagulants.

\section{METHODS}

Retrospective cohort study using data from the National Swedish Patient Register, Dispensed Drug Register, Cause of Death Register and the socioeconomic longitudinal integration database for health insurance and labour market studies (LISA) register.

For the identification of patients and record linkage, we used the individual civic registration numbers that are given to all permanent residents in Sweden irrespective of citizenship. These numbers are used in all contacts with healthcare including pharmacies across the country and make it possible to follow each individual's medical history over a lifetime. Opting out is not possible.

The Swedish Patient Register carries information about all hospitalisations in the country since 1987 with information about codes for diagnoses, surgery, investigative procedures and trauma. Laboratory values and results of examinations are not available in the register.

The Drug Register stores details about every dispensed prescription in Sweden since 1 July 2005. All pharmacies in the country are required to participate by law, and information is transferred electronically whenever a drug is dispensed. The Drug Register does not contain information about prescriptions which has not been dispensed, drugs used during hospital stay or over-thecounter drugs.

The LISA register carries detailed information about, for example, educational level, occupation, income, immigrant status and marital status.

The Cause of Death Register carries detailed information about deaths, with at least one underlying cause of death and up to 48 contributing causes of death.
These Swedish registers have frequently been used for epidemiological and outcome studies and the general quality of data is good according to validation studies. ${ }^{20-23}$

\section{Identification of the study population}

A new-user retrospective cohort was defined as all individuals aged $\geq 18$ years with atrial fibrillation who had filled their first prescription for apixaban, dabigatran, rivaroxaban or warfarin during the study period between 1 December 2011 and 31 December 2014. The study starting date was chosen based on the date when the first NOAC dabigatran was made available for general prescription and reimbursed for stroke prevention in atrial fibrillation in Sweden. Rivaroxaban was available from October 2012 and apixaban from May 2013, while edoxaban was not available for stroke prevention in atrial fibrillation during the study period. The study period and the observation period were the same; thus, minimum follow-up was 1 day for the latest included patient.

The first filled prescription for NOAC or warfarin during the study period defined index date. Patients who had received any oral anticoagulant before the index prescription were excluded in order to make sure that only oral anticoagulation naïve patients were included; this lookback period for previous oral anticoagulation drug use goes back to 1 July 2005 when the Swedish Drug Register was started, thus at a minimum 6 years and 5 months for patients with earliest index dates in this study. Patients with mitral stenosis or mechanical valvular replacement prosthesis were excluded as NOACs are neither approved nor recommended for these patients. ${ }^{24}$

\section{Baseline characterisation of study groups}

The Patient Register was also used for characterisation of previous and concomitant diseases for each subject at inclusion in the study. Unless otherwise specified, registry information from 1997 up to and including index date was used. The definitions, according to International Classification of Diseases, 10th edition (ICD-10) codes, are presented in online supplementary table 1 . Other medication at baseline was defined by filled prescriptions made within 6 months before index in the Drug Register.

\section{Follow-up and study outcomes}

Time at risk was counted from the day of the first purchase of respective anticoagulant. Subjects were monitored until first purchase of another oral anticoagulant, first outcome event or were censored at the end of the study period.

The Patient Register and the Cause of Death Register were used for identification of safety and efficacy outcome events, defined by ICD-10 codes. Although the Patient Register also holds information about outpatient visits, only diagnoses given in conjunction with hospitalisation or death were used to define outcome events. For ischaemic stroke, systemic embolism and myocardial infarction, only the principal and the first secondary diagnoses were considered. For major bleeding events, all principal 
and secondary diagnoses were considered, in accordance with the results of a recent Swedish validation study. ${ }^{25}$ ICD-10 codes for the study endpoints are listed in online supplementary table 2 .

\section{Statistical methods}

Baseline characteristics are presented descriptively and differences were tested with Wilcoxon-Mann-Whitney's rank sum test and Pearson's $\mathrm{X}^{2}$ test. Incidence rates were calculated as events per 100 person-years at risk for each outcome.

Propensity scores for the likelihood of treatment with an NOAC rather than warfarin were obtained by logistic regression with the following independent variables: NOAC treatment; female sex; age (continuous); married or cohabitating; immigrant status; higher education $(>12$ years); highest income quartile; newly diagnosed atrial fibrillation; any previous intracranial haemorrhage; any other major bleeding event; anaemia; coagulation defect; renal failure; liver disease; previous ischaemic stroke; heart failure; hypertension; diabetes; previous myocardial infarction; valvular disease other than mitral stenosis or mechanical heart valve; implanted pacemaker or implantable cardioverter defibrillator; hyperthyroidism in the previous year; previous hypothyroidism; chronic obstructive pulmonary disease; asthma; previous pulmonary embolism; cancer diagnosis within the previous 3years; an alcohol index diagnosis; dementia; use of non-steroidal anti-inflammatory drug, acetylsalicylic acid or clopidogrel at study inclusion. These individual propensity scores were used as a continuous covariate in the multivariable analyses.

Multivariable competing risk regression of sub-HRs accounting for the risk of dying before reaching specified outcomes was done according to the method of Fine and $\mathrm{Gray}^{26}$ to evaluate the association between outcomes and treatment regime, and multivariable Cox regression to evaluate the association between mortality and treatment regime. The covariates used are presented in a footnote to figure 1 . We investigated potential violations against the proportional hazards assumption graphically and using Schoenfeld residuals but detected no problems with any of the endpoints.

The number of days from inclusion to the end of the study period on 31 December 2014 was used as a covariate. The rationale for this is to capture confounding from temporal effects, for example, that drugs with a shorter mean observation period (ie, NOACs) are likely to have more complications than a drug that has been used for a

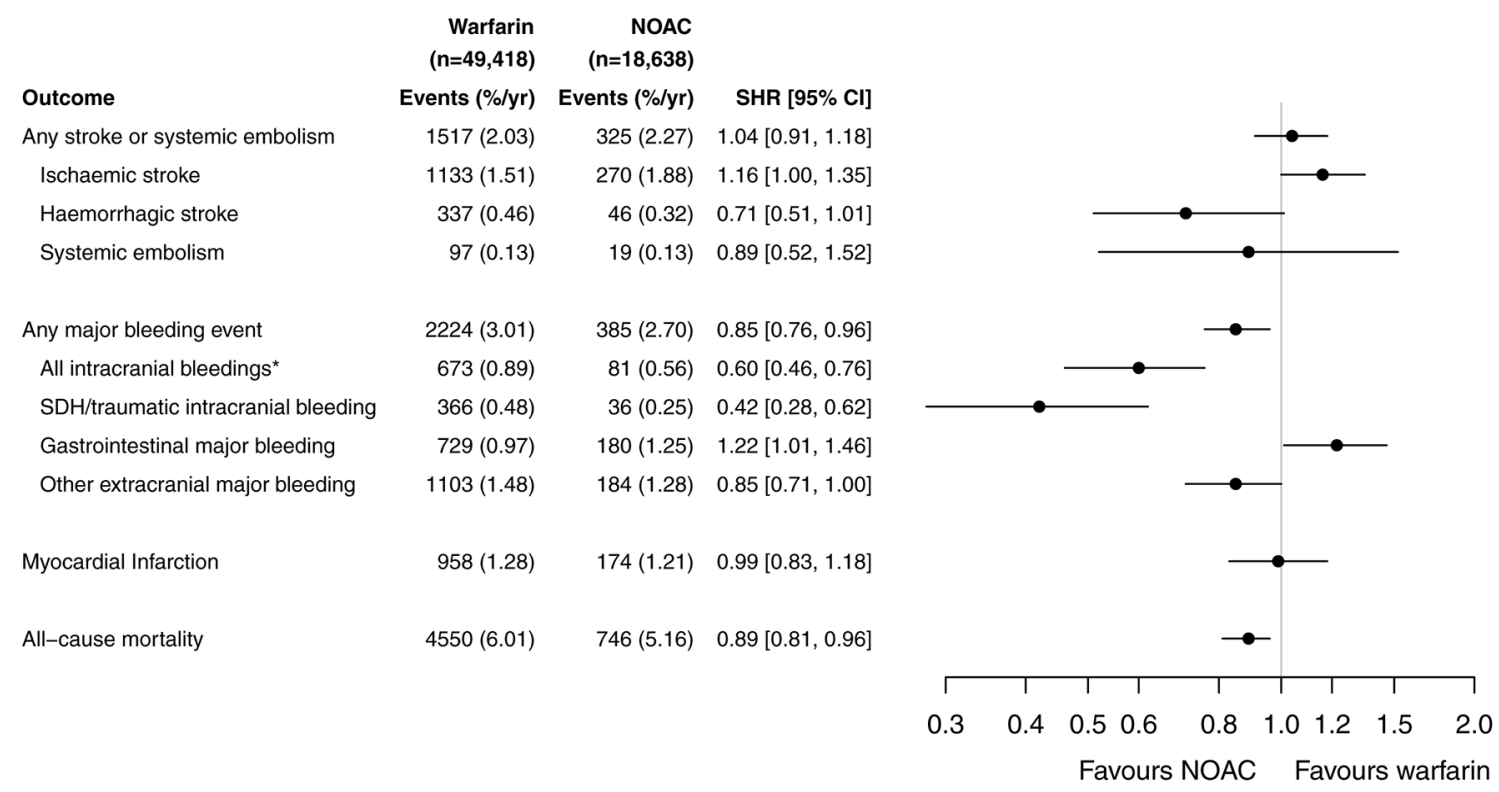

Figure 1 Efficacy and safety outcomes with NOAC compared with warfarin in 18638 NOAC patients with 14457 years of risk and 49418 warfarin patients with 75747 person-years at risk. Multivariable Fine-Gray competing risks models were used to evaluate the association between outcomes and treatment regime, accounting for the competing risk of death for all outcomes except all-cause mortality. Multivariable Cox regression was used to evaluate the association between mortality and treatment regime. Multivariable adjustments for age (continuous), gender, marital status, immigrant status, higher education ( $>12$ years), income in the top quartile, first atrial fibrillation diagnosis within 3 months before index, intracranial bleed, gastrointestinal bleed, urogenital bleed, other bleed, anaemia, coagulation or platelet defect, renal failure, liver disease, ischaemic stroke, heart failure, hypertension, diabetes, myocardial infarction, valvular disease (other than exclusion criteria), pacemaker/ implantable cardioverter defibrillator, thyrotoxicosis with preceding years, hypothyroidism, chronic obstructive pulmonary disease, asthma, pulmonary embolism, cancer within 3 years, alcohol index, hospitalisation for fall accident $\geq 2$ times, dementia, baseline use of aspirin, clopidogrel, or non-steroidal anti-inflammatory drugs, days from index to 31 December 2014 and propensity score for likelihood of receiving NOACs rather than warfarin treatment. *Intracranial bleeding events consists of haemorrhagic stroke, subdural haematomas and traumatic intracranial bleeding. NOAC, non-vitamin K antagonist oral anticoagulant; SDH, subdural haematoma; SHR, sub-HR. 
longer time (ie, warfarin) because complications due to treatment are more common shortly after initiation.

Statistical significance was considered when $p$ values $<0.05$. All analyses were performed using Stata V.14.0 (Stata).

The study was approved by the Stockholm regional ethics committee (approvals \#2014/894-31, \#2014/87631/4, \#2014/1065-31) and conformed to the Declaration of Helsinki.

\section{RESULTS}

During the 37-month inclusion period, 68056 new users of anticoagulants who fulfilled the study criteria were identified: 49418 on warfarin and 18638 on NOAC treatment (apixaban 6547 (33.3\% on reduced dose), dabigatran $6651(33.5 \%$ on $110 \mathrm{mg}$ dose) and 5440 rivaroxaban (32.4\% on reduced dose)). For patient flow chart, see figure 2. The median follow-up for the mortality outcome was 0.71 years in the NOAC group and 1.74 years in the warfarin group, $\mathrm{p}<0.001$.

\section{Baseline characteristics}

Baseline patient characteristics are presented in table 1. The proportions of men and women were similar with NOACs and warfarin; mean age was also similar (73.4 vs 73.7 years). Within the NOAC group, dabigatran patients were younger (70.2 years) than patients on apixaban (75.0 years) and rivaroxaban (75.1 years).

The proportion of individuals with higher education was higher with NOACs than with warfarin $(25.7 \%$ vs $20.2 \%)$. The mean income was also higher with NOACs than with warfarin.

The mean $\mathrm{CHA}_{2} \mathrm{DS}_{2}$-VASc score was lower in the NOACs than in the warfarin cohort (3.24 vs 3.38 points). Within the NOAC cohort, the proportion of patients with a score of 0 or 1 was higher with dabigatran $(25.1 \%)$ than with rivaroxaban $(12.9 \%)$ and apixaban $(13.8 \%)$. The proportion of patients who were cardioverted during follow-up was $23.3 \%$ with dabigatran, $19.5 \%$ with warfarin, $7.9 \%$ with apixaban and $5.8 \%$ with rivaroxaban.

Patients on NOACs had a longer history of atrial fibrillation as measured from the very first diagnosis of atrial fibrillation in the Patient Register (mean 2.48 vs 1.72 years). The proportion with prior thromboembolic and major bleeding events were similar among NOAC-treated patients than with warfarin-treated patients, as well as several concomitant diseases including hypertension. Heart failure, diabetes mellitus, renal failure and history of myocardial infarction were more common in patients on warfarin.

\section{Outcomes}

Outcomes are presented in figure 1 . The unadjusted incidence of the composite of all-cause stroke or systemic embolism was similar with NOACs and warfarin $(2.27 \%$ vs $2.03 \%$ per year). After adjustment for cofactors and for the competing risk of death, there were no statistically
Table 1 Baseline patient characteristics

\begin{tabular}{llll}
\hline & $\begin{array}{l}\text { All } \\
(\mathbf{n = 6 8}\end{array}$ & $\begin{array}{l}\text { NOAC } \\
(\mathbf{n = 1 8} \\
\text { Variable }\end{array}$ & $\begin{array}{l}\text { Warfarin } \\
(\mathbf{n = 4 9}\end{array}$ \\
\hline Age, mean (median) & $73.6(74)$ & $73.4(74)$ & $73.7(75)$ \\
\hline Women & 30714 & $45.6 \%$ & $45.0 \%$ \\
\hline Married/cohabitant & 36192 & $52.9 \%$ & $53.3 \%$ \\
\hline Immigrant & 7623 & $11.5 \%$ & $11.1 \%$ \\
\hline Higher education (>12 years) & 14753 & $25.7 \%$ & $20.2 \%$ \\
\hline Income, mean* (median) & $216(165)$ & $232(172)$ & $209(163)$ \\
\hline $\begin{array}{l}\text { Years since first AF diagnosis, } \\
\text { mean (median) }\end{array}$ & $1.93(0.04)$ & $2.48(0.08)$ & $1.72(0.03)$ \\
\hline $\begin{array}{l}\text { CHA }{ }_{2} \text { DS } \\
\text { (median) }\end{array}$ & $3.34(3)$ & $3.24(3)$ & $3.38(3)$ \\
\hline
\end{tabular}

\begin{tabular}{|llll}
\hline Bleeding history & & & \\
\hline Any major bleeding & 6659 & $10.2 \%$ & $9.6 \%$ \\
\hline Intracranial & 882 & $1.6 \%$ & $1.2 \%$ \\
\hline Gastrointestinal & 2210 & $3.4 \%$ & $3.2 \%$ \\
\hline Other major bleeding & 4236 & $6.4 \%$ & $6.2 \%$ \\
\hline
\end{tabular}

\section{Thromboembolic history}

\begin{tabular}{|c|c|c|c|}
\hline Ischaemic stroke & 8838 & $13.4 \%$ & $12.8 \%$ \\
\hline Unspecified stroke & 423 & $0.7 \%$ & $0.6 \%$ \\
\hline Systemic embolism & 605 & $0.7 \%$ & $1.0 \%$ \\
\hline Transient ischaemic attack & 4128 & $6.1 \%$ & $6.1 \%$ \\
\hline Pulmonary embolism & 1867 & $1.9 \%$ & $3.1 \%$ \\
\hline Anaemia & 6267 & $8.8 \%$ & $9.4 \%$ \\
\hline Coagulation or platelet defect & 688 & $0.9 \%$ & $1.1 \%$ \\
\hline Renal failure & 2768 & $2.5 \%$ & $4.7 \%$ \\
\hline Liver disease & 866 & $1.4 \%$ & $1.2 \%$ \\
\hline Heart failure & 14942 & $19.5 \%$ & $22.9 \%$ \\
\hline Hypertension & 41889 & $61.1 \%$ & $61.7 \%$ \\
\hline Diabetes & 12253 & $16.1 \%$ & $18.7 \%$ \\
\hline Myocardial infarction & 9150 & $10.8 \%$ & $14.4 \%$ \\
\hline Valvular disease $\dagger$ & 6515 & $7.6 \%$ & $10.3 \%$ \\
\hline $\begin{array}{l}\text { Pacemaker/Implantable } \\
\text { cardioverter defibrillator }\end{array}$ & 5055 & $8.2 \%$ & $7.1 \%$ \\
\hline $\begin{array}{l}\text { Thyrotoxicosis within preceding } \\
\text { year }\end{array}$ & 499 & $0.7 \%$ & $0.8 \%$ \\
\hline Hypothyroidism & 4642 & $6.9 \%$ & $6.8 \%$ \\
\hline $\begin{array}{l}\text { Chronic obstructive pulmonary } \\
\text { disease }\end{array}$ & 5002 & $6.8 \%$ & $7.6 \%$ \\
\hline Asthma & 3924 & $5.9 \%$ & $5.7 \%$ \\
\hline $\begin{array}{l}\text { Cancer within preceding } \\
3 \text { years }\end{array}$ & 6043 & $8.6 \%$ & $9.0 \%$ \\
\hline Alcohol indexł & 2023 & $3.3 \%$ & $2.9 \%$ \\
\hline Dementia & 1031 & $1.9 \%$ & $1.4 \%$ \\
\hline $\begin{array}{l}\text { Hospitalised for fall } \\
\text { accidents } \geq 2 \text { times }\end{array}$ & 2872 & $4.8 \%$ & $4.0 \%$ \\
\hline
\end{tabular}

Continued 


\begin{tabular}{llll}
\hline Table 1 & Continued & & \\
\hline & $\begin{array}{l}\text { All } \\
(\mathbf{n}=68\end{array}$ & $\begin{array}{l}\text { NOAC } \\
(\mathbf{n}=\mathbf{1 8}\end{array}$ & $\begin{array}{l}\text { Warfarin } \\
(\mathbf{n}=\mathbf{4 9}\end{array}$ \\
Variable & $\mathbf{0 5 6 )}$ & $\mathbf{6 3 8 )}$ & $\mathbf{4 1 8 )}$ \\
\hline $\begin{array}{l}\text { Aspirin, clopidogrel or NSAID } \\
\text { at index }\end{array}$ & 9572 & $12.7 \%$ & $14.6 \%$ \\
\hline
\end{tabular}

*Disposable annual income or pension in thousands of Swedish crowns, after taxes and related to number of persons in the household.

tOther valvular disease than what is counted as exclusion criteria, for example, mostly mitral regurgitation, aortic sclerosis and/or stenosis common among elderly subjects.

$\ddagger$ Set of diagnostic codes used by the Swedish Board of Health and Welfare for reporting of alcohol-related mortality. See appendix.

$\mathrm{AF}$, atrial fibrillation; NOAC, non-vitamin $\mathrm{K}$ antagonist oral anticoagulant; NSAID, non-steroidal anti-inflammatory drug.

significant differences in all-cause stroke or systemic embolism (sub-HR (SHR) 1.04, 95\% CI(0.91 to 1.18) (figure 2).

The unadjusted incidence of ischaemic stroke was not statistically significantly different with NOACs and warfarin $(1.88 \%$ vs $1.51 \%$ per year). After adjustments, there was a $16 \%$ higher risk for ischaemic stroke with NOACs than with warfarin at borderline statistical significance (SHR 1.16, CI 1.00 to 1.35).

The unadjusted incidence of major bleeding events was lower with NOACs than with warfarin $(2.70 \%$ vs $3.01 \%$ per year). After adjustments, a statistically significant $15 \%$ lower risk of major bleeds with NOACs than with warfarin was seen (SHR 0.85, CI 0.76 to 0.96).

The incidence of intracranial bleeding was higher with warfarin than with NOACs $(0.89 \%$ vs $0.56 \%$ per year). After adjustments, the risk was $40 \%$ lower with NOACs than with warfarin (SHR 0.60, CI 0.46 to 0.76 ).

When the analysis was confined to haemorrhagic stroke, that is intracerebral and subarachnoidal bleeding events, which constituted a little less than half of all intracranial bleeding events, the apparent benefit of NOACs did not quite reach statistical significance (SHR 0.71, CI 0.51 to 1.01 ). The apparent benefit of NOACs was larger regarding the composite of subdural haematomas and traumatic intracranial bleeding events (SHR 0.42, CI 0.28 to 0.62 ).

Gastrointestinal bleeds were more frequent with NOACs than with warfarin $(1.25 \%$ vs $0.97 \%$ per year $)$. After multivariable adjustments, an excess risk of $22 \%$ with NOACs rather than with warfarin remained (SHR 1.22, CI 1.01 to 1.46). However, other extracranial major bleeding events, mostly urogenital, were less frequent with NOAC than with warfarin (SHR 0.85 , CI 0.71 to $1.00)$.

The unadjusted incidence of all-cause mortality was lower with NOACs than with warfarin $(5.16 \%$ vs $6.01 \%$ per year). After adjustments, a statistically significant survival benefit was seen with NOACs relative to warfarin (HR 0.89, CI 0.81 to 0.96 ).

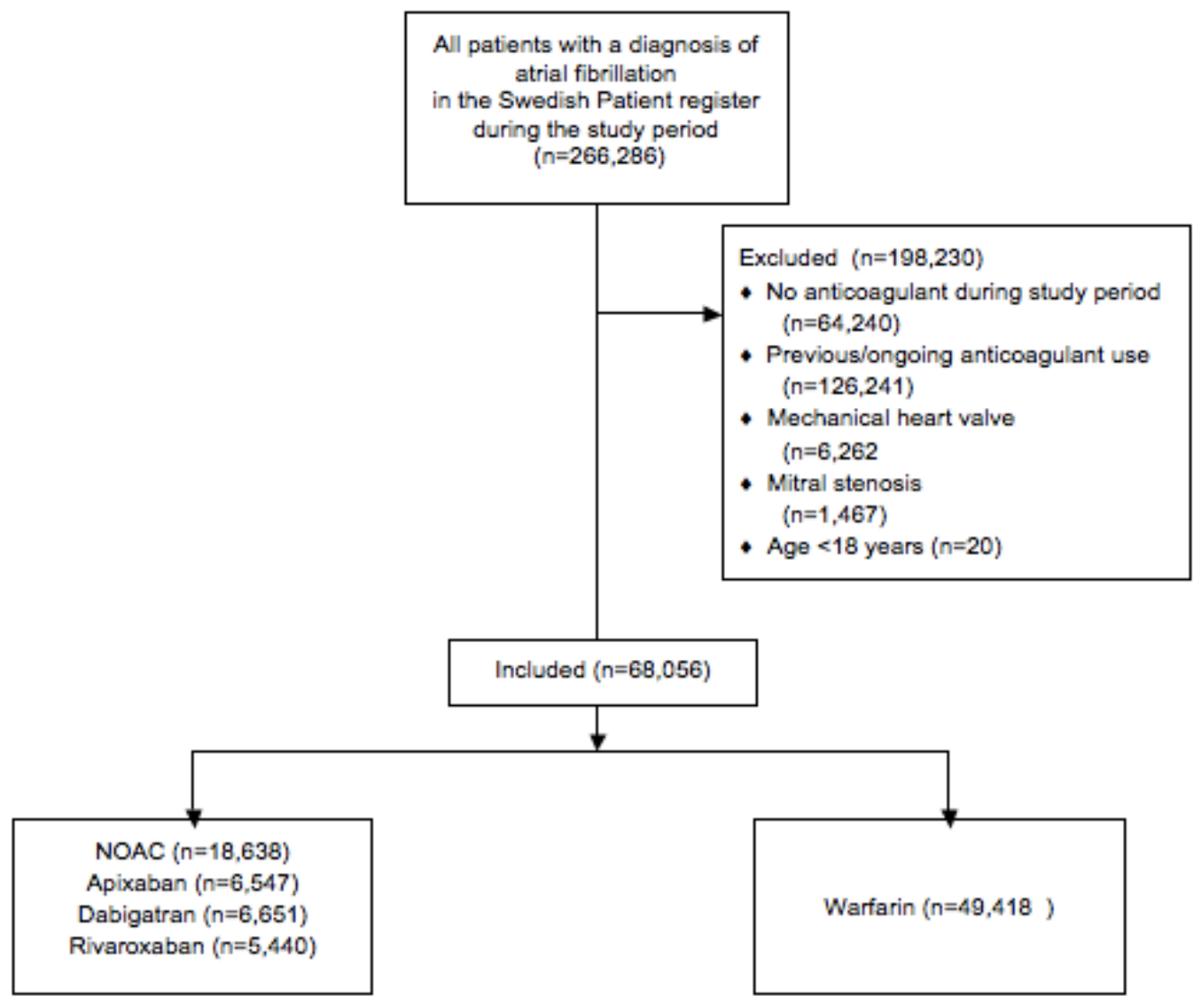

Figure 2 Patient flow chart. NOAC, non-vitamin $\mathrm{K}$ antagonist oral anticoagulant. 


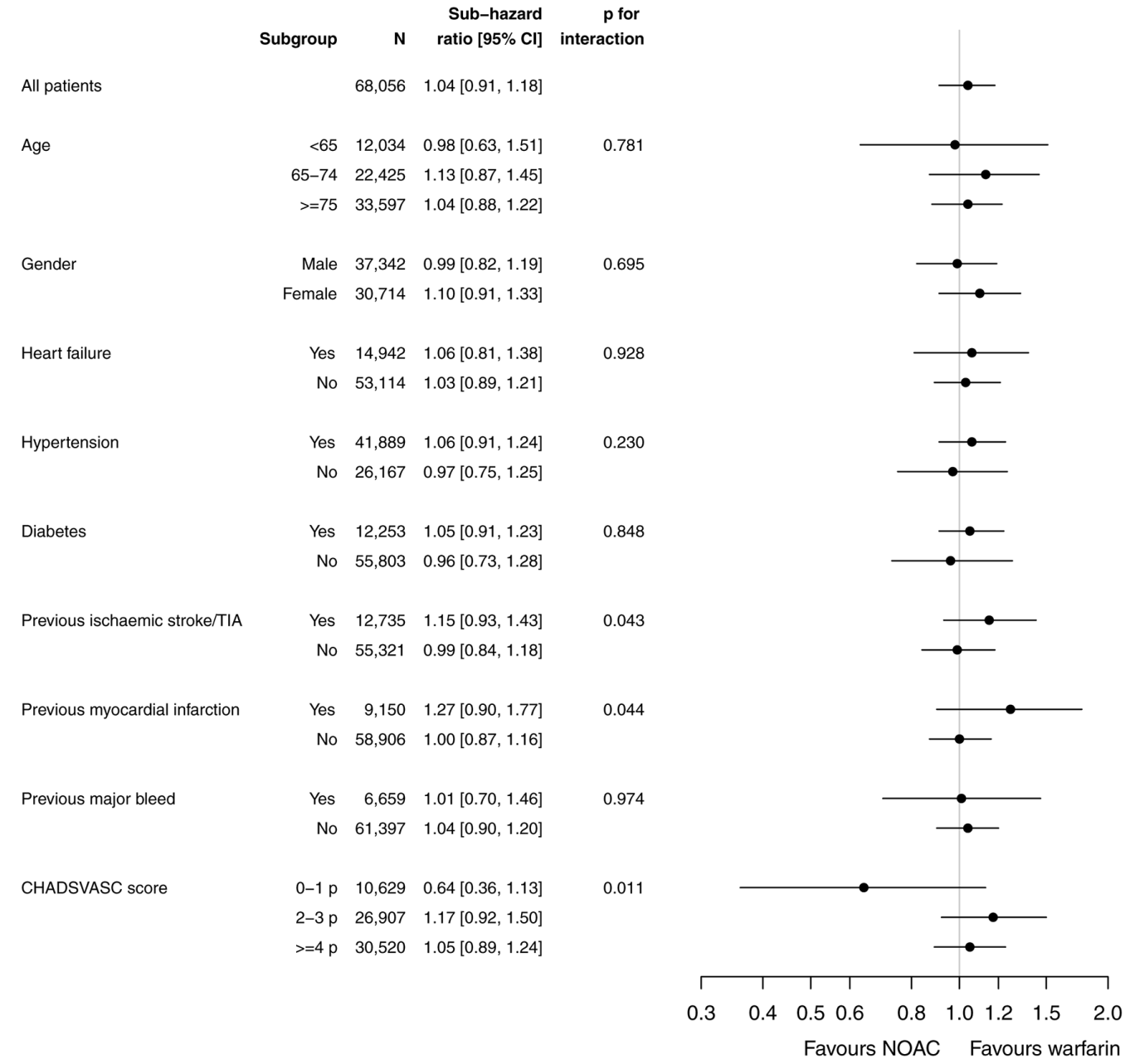

Figure 3 Subgroup analyses of all-cause stroke or systemic embolism, NOAC compared with warfarin. NOAC, non-vitamin K antagonist oral anticoagulant; TIA, transient ischaemic attack.

The incidences of myocardial infarction and systemic embolism were similar between the treatment groups.

\section{Subgroup analyses}

All-cause stroke and systemic embolism were similar across many subgroups, with a statistically significant interaction indicating potential benefit of NOACs in patients with $\mathrm{CHA}_{2} \mathrm{DS}_{2}$-VASc score 0-1 (figure 3).

A reduction in risk of major bleeding events with NOACs compared with warfarin was consistent across several subgroups, but with interactions indicating greater reduction with NOACs compared with warfarin in patients younger than 65 years, those with $\mathrm{CHA}_{2} \mathrm{D}_{\mathrm{s}}$-VASc scores $0-1$ and without a history of myocardial infarction (figure 4).

A reduction in mortality was consistent across most subgroups, but less pronounced in NOAC patients with a history of stroke or transient ischaemic attack (figure 5).

\section{DISCUSSION}

In this large non-selected anticoagulation-naïve Swedish atrial fibrillation cohort starting oral anticoagulation treatment, the incidence of all-cause stroke or systemic embolism did not statistically differ between patients receiving NOAC or well-managed warfarin treatment.
NOAC use was associated with lower risks of all-cause mortality, major bleeding, haemorrhagic stroke and intracranial haemorrhage, but with higher risks of gastrointestinal bleeding events.

Educational level and income was higher with NOACs than with warfarin. Patient cost probably played a minor role because Swedish subsidies prevent patients from having to spend more than 2200 SEK (€244) for medicines annually and the low price of warfarin is counterbalanced by patient's costs for regular INR controls. Thus, the cost to patients is neutral.

The safety results of this study, that is, significantly fewer major bleeding events except for gastrointestinal bleeding, and significantly lower mortality, with NOACs than with warfarin were similar in direction and magnitude to both the meta-analysis of the four pivotal RCTs of NOAC versus warfarin, ${ }^{10}$ and the to date largest published real-world data set of NOAC compared with VKA consisting of $>134000$ elderly Medicare patients ${ }^{27}$ in the USA treated with dabigatran or warfarin.

There was no reduction in all-cause stroke or systemic embolism with NOACs compared with warfarin in contrast to the statistically significant although modest 


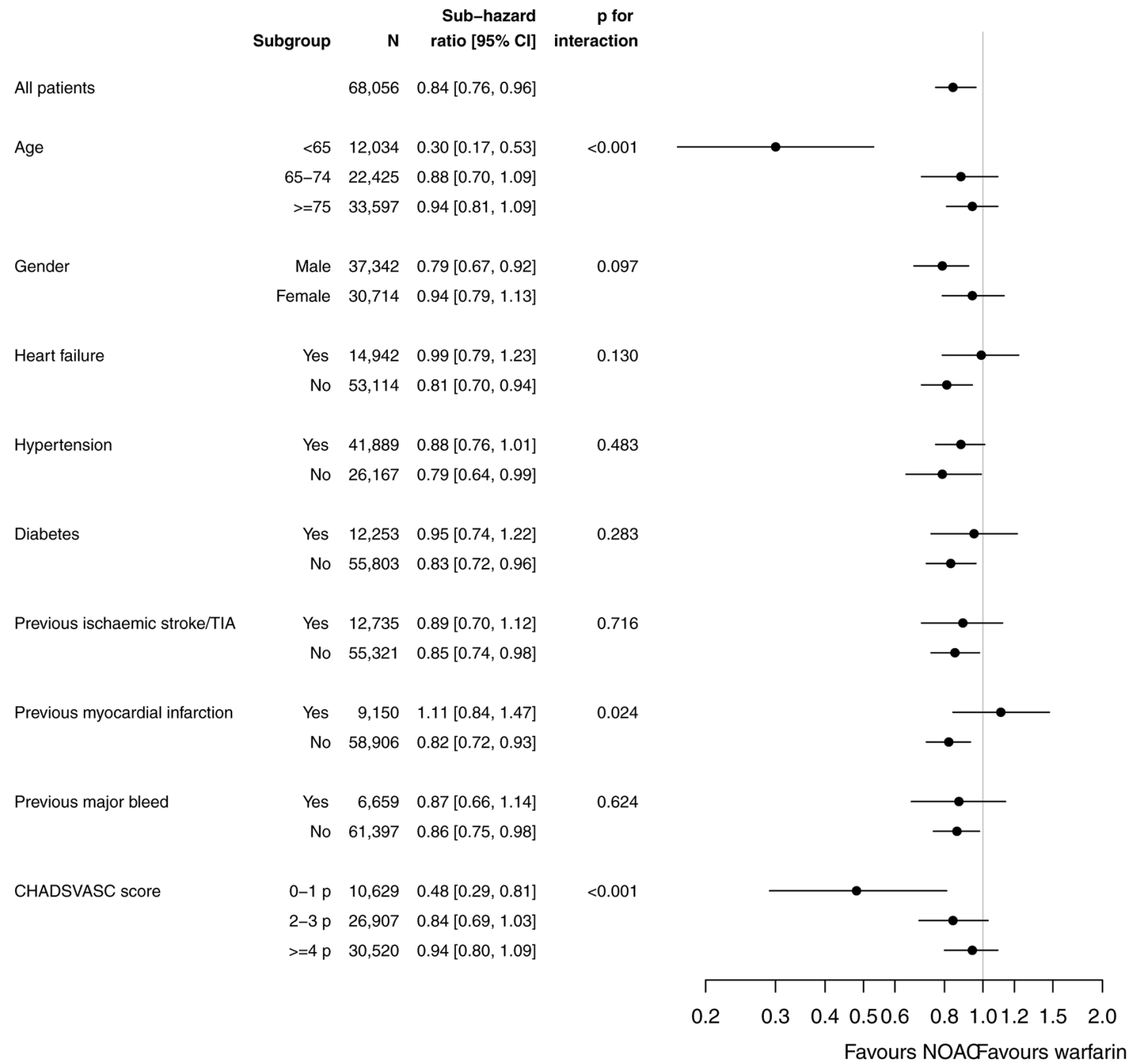

Figure 4 Subgroup analyses of major bleeding events, NOAC compared with warfarin. NOAC, non-vitamin K antagonist oral anticoagulant; TIA, transient ischaemic attack.

reduction in the aforementioned RCT meta-analysis. ${ }^{10}$ There was even a trend for higher risk for ischaemic stroke with NOACs versus warfarin after adjustments including competing risks for death, the latter significantly reduced by NOACs. This result might reflect the fact that warfarin treatment in Sweden generally is of much higher standard than in the pivotal NOAC trials. ${ }^{18} 19$ Similar results on stroke/thromboembolism and ischaemic stroke, respectively, have recently been shown in a cohort study from Denmark, ${ }^{28}$ where warfarin treatment is also of high standard. Ischaemic stroke was neither reduced by NOACs compared to warfarin in the RCT meta-analysis, ${ }^{10}$ the latter based on the outcomes of the original four RCTs where only dabigatran $150 \mathrm{mg}$ was shown to reduce ischaemic stroke. ${ }^{6-9}$

Subgroup analyses should be cautiously interpreted, especially regarding all-cause stroke or systemic embolism where the main results showed no difference between NOACs and warfarin. The reduction in major bleeding events and mortality by NOACs compared with warfarin seemed more pronounced in younger patients at lower stroke risk. Importantly, neither the elderly, patients at higher stroke risk, nor any other subgroup appeared to benefit more from warfarin than from NOACs for any of those safety outcomes.

A substantially higher risk for intracranial haemorrhage and haemorrhagic strokes with warfarin than NOAC has consistently been shown. ${ }^{6-9}$ The present finding of a pronounced reduction in subdural haematomas and traumatic intracranial bleeding events is intriguing. A potential explanation is the different effects on coagulation system. ${ }^{29}$ At sites of cerebral vascular injury, exposed tissue factor induces thrombin generation that can overcome the inhibitory effects of NOACs whereas the markedly reduced levels of FVIIa by warfarin will impair this response and increase the likelihood of a severe bleeding even by a modest trauma to the head, although this hypothesis remains to be proven and further studies exploring traumatic intracranial bleedings in patients with oral anticoagulants are warranted. 


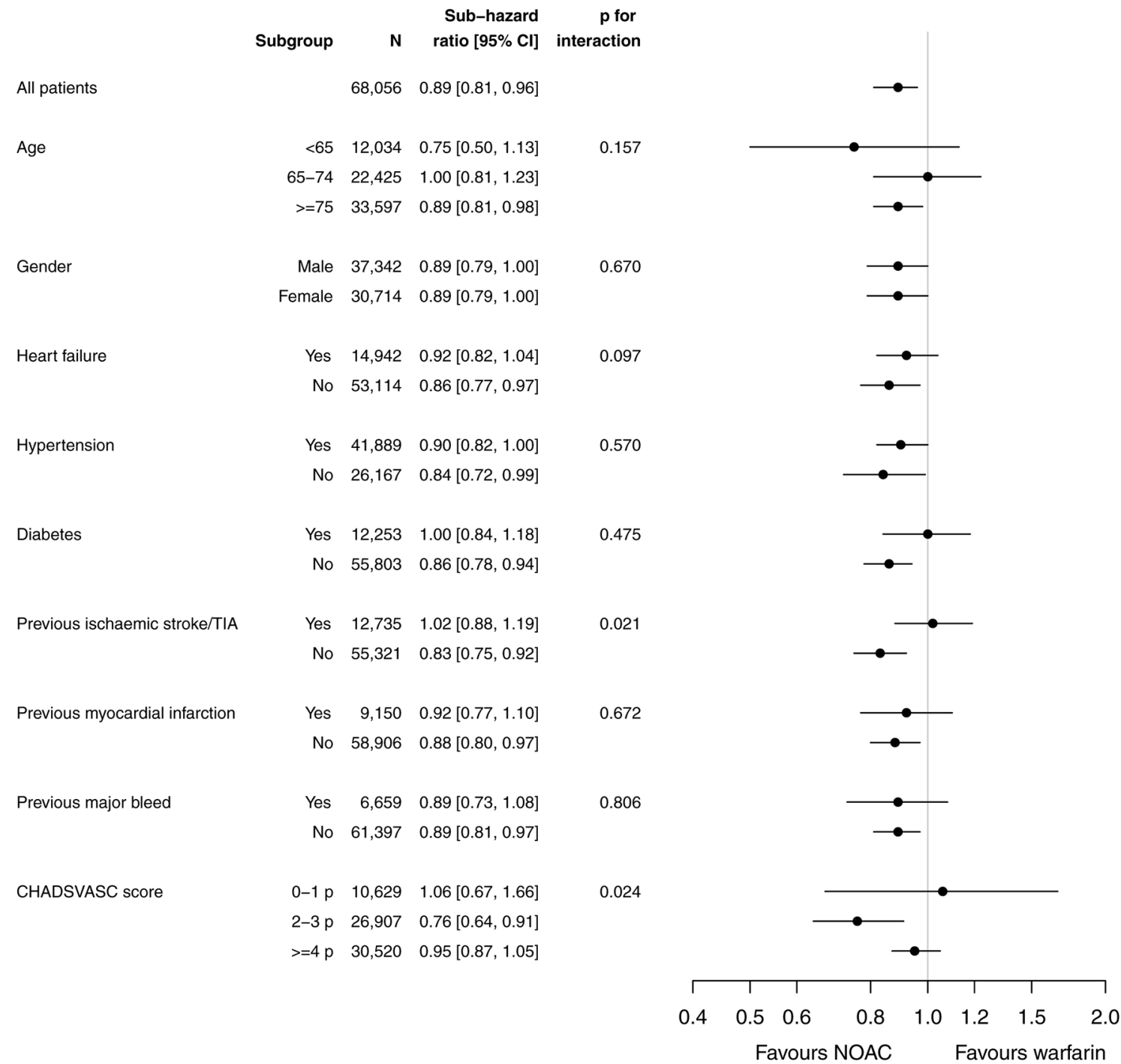

Figure 5 Subgroup analyses of all-cause mortality, NOAC compared with warfarin. NOAC, non-vitamin K antagonist oral anticoagulant; TIA, transient ischaemic attack.

\section{Limitations and strengths}

This study has limitations. It was observational and may, despite propensity scores and adjustment for an extensive number of variables and competing risks, be subject to residual confounding from factors not adjusted for in the analysis. Thus, we refrained from analyses of the smaller groups of each individual NOAC versus warfarin where the risk for residual confounding was deemed even higher.

The databases did not include laboratory results; therefore, we could not assess anaemia, renal function (eg, for evaluation of proper dose reductions of NOACs) or the quality of warfarin treatment. However, results from Swedish patients enrolled in clinical trials ${ }^{1617}$ as well non-selected patients in general practice or at anticoagulation clinics, ${ }^{18} 19$ have repeatedly shown mean TTR above the recommended $70 \%$ and we have no reason to assume otherwise in this study cohort. Furthermore, in the meta-analysis of the randomised controlled NOACs versus warfarin trials, ${ }^{10}$ there was a suggestion of even greater relative reduction in bleeding with NOACs at centres with poor INR control. This would, if anything, suggest an underestimation of the improved safety by NOACs versus warfarin in this study if TTR would be less than in previously published Swedish results.

Strengths include the large contemporary sample rendering more than 9000 outcome events, universal coverage of the prescription registry, and hence inclusion of all oral anticoagulation users nationwide, the universal coverage of the high-precision ${ }^{20}$ registries for determining the outcomes and no loss to follow-up. Although the total number of outcome events was high, there was limited possibility for subgroup analysis of separate outcomes such as ischaemic stroke, haemorrhagic stroke or myocardial infarction. Another strength is that the warfarin and NOAC cohorts were rather similar regarding age, gender, history of thromboembolic and bleeding events from the outset. We nevertheless used propensity scores and multivariate regression to adjust for differences on observed variables and follow-up between the treatment groups. 


\section{CONCLUSION}

In this large non-selected Swedish atrial fibrillation cohort without previous oral anticoagulation treatment, NOACs were no better than well-managed warfarin for prevention of all-cause stroke or systemic embolism. NOAC use was associated with lower risks of all-cause mortality, major bleeding and intracranial haemorrhage, but with higher gastrointestinal bleeding risk. The substantially improved safety and lower mortality with NOACs compared with warfarin suggests NOACs as the preferred treatment for patients with atrial fibrillation starting oral anticoagulation for stroke prevention.

Acknowledgements Editorial support was provided by Ebba Bergman, $\mathrm{PhD}$, and Vendela Roos, PhD, UCR, Uppsala, Sweden.

Contributors Both authors have made substantial contributions to the conception or design of the work; the acquisition, analysis and interpretation of data for the work; and drafting the work and revising it critically for important intellectual content; and final approval of the original and revised version to be published; and agree to be accountable for all aspects of the work in ensuring that questions related to the accuracy or integrity of any part of the work are appropriately investigated and resolved.

Funding No other parties than the authors themselves has participated or influenced study design, analyses or interpretation of results. All authors had full access to all the data in the study and take full responsibility for the integrity of the data and accuracy of the data analysis.

Competing interests Outside of the present work, LF has received funding from or given lectures for Bayer, Boehringer Ingelheim, Bristol Myers Squibb, Pfizer, Sanofi and St Jude Medical. J0 reports advisory board and lecture fees from Bayer, Boehringer Ingelheim, Bristol-Myers Squibb, Pfizer and Sanofi.

Ethics approval Stockholm regional ethics committee.

Provenance and peer review Not commissioned; internally peer reviewed.

Open Access This is an Open Access article distributed in accordance with the Creative Commons Attribution Non Commercial (CC BY-NC 4.0) license, which permits others to distribute, remix, adapt, build upon this work non-commercially, and license their derivative works on different terms, provided the original work is properly cited and the use is non-commercial. See: http://creativecommons.org/ licenses/by-nc/4.0/

(c) Article author(s) (or their employer(s) unless otherwise stated in the text of the article) 2017. All rights reserved. No commercial use is permitted unless otherwise expressly granted.

\section{REFERENCES}

1. Hart RG, Pearce LA, Aguilar MI. Meta-analysis: antithrombotic therapy to prevent stroke in patients who have nonvalvular atrial fibrillation. Ann Intern Med 2007;146:857-67.

2. Själander A, Engström G, Berntorp E, et al. Risk of haemorrhagic stroke in patients with oral anticoagulation compared with the general population. J Intern Med 2003;254:434-8.

3. Hylek EM, Go AS, Chang Y, et al. Effect of intensity of oral anticoagulation on stroke severity and mortality in atrial fibrillation. $N$ Engl J Med 2003;349:1019-26.

4. Reynolds MW, Fahrbach K, Hauch O, et al. Warfarin anticoagulation and outcomes in patients with atrial fibrillation: a systematic review and metaanalysis. Chest 2004;126:1938-45.

5. White HD, Gruber M, Feyzi J, et al. Comparison of outcomes among patients randomized to warfarin therapy according to anticoagulant control: results from SPORTIF III and V. Arch Intern Med 2007;167:239-45.

6. Connolly SJ, Ezekowitz MD, Yusuf S, et al. Dabigatran versus warfarin in patients with atrial fibrillation. $N$ Engl $J$ Med 2009;361:1139-51.
7. Patel MR, Mahaffey KW, Garg J, et al. Rivaroxaban versus warfarin in nonvalvular atrial fibrillation. N Engl J Med 2011;365:883-91.

8. Granger $\mathrm{CB}$, Alexander JH, McMurray JJ, et al. Apixaban versus warfarin in patients with atrial fibrillation. $N$ Engl $\mathrm{J} \mathrm{Med}$ 2011;365:981-92.

9. Giugliano RP, Ruff CT, Braunwald E, et al. Edoxaban versus warfarin in patients with atrial fibrillation. N Engl J Med 2013;369:2093-104.

10. Ruff CT, Giugliano RP, Braunwald E, et al. Comparison of the efficacy and safety of new oral anticoagulants with warfarin in patients with atrial fibrillation: a meta-analysis of randomised trials. Lancet 2014;383:955-62.

11. De Caterina R, Husted S, Wallentin L, et al. Vitamin K antagonists in heart disease: current status and perspectives (Section III). Position paper of the ESC Working Group on Thrombosis-Task Force on Anticoagulants in Heart Disease. Thromb Haemost 2013;110:1087-107.

12. Björck F, Renlund H, Svensson PJ, et al. Warfarin persistence among stroke patients with atrial fibrillation. Thromb Res 2015;136:744-8.

13. Baker WL, Cios DA, Sander SD, et al. Meta-analysis to assess the quality of warfarin control in atrial fibrillation patients in the United States. J Manag Care Pharm 2009;15:244-52.

14. Gallagher AM, Setakis E, Plumb JM, et al. Risks of stroke and mortality associated with suboptimal anticoagulation in atrial fibrillation patients. Thromb Haemost 2011;106:968-77.

15. Oldgren J, Healey JS, Ezekowitz M, et al. Variations in cause and management of atrial fibrillation in a prospective registry of 15,400 emergency department patients in 46 countries: the RE-LY Atrial Fibrillation Registry. Circulation 2014;129:1568-76.

16. Connolly SJ, Pogue J, Eikelboom J, et al. Benefit of oral anticoagulant over antiplatelet therapy in atrial fibrillation depends on the quality of international normalized ratio control achieved by centers and countries as measured by time in therapeutic range. Circulation 2008;118:2029-37.

17. Wallentin L, Yusuf S, Ezekowitz MD, et al. Efficacy and safety of dabigatran compared with warfarin at different levels of international normalised ratio control for stroke prevention in atrial fibrillation: an analysis of the RE-LY trial. Lancet 2010;376:975-83.

18. Sjögren V, Grzymala-Lubanski B, Renlund $\mathrm{H}$, et al. Safety and efficacy of well managed warfarin. A report from the Swedish quality register Auricula. Thromb Haemost 2015;113.

19. Björck $F$, Sandén $P$, Renlund $H$, et al. Warfarin treatment quality is consistently high in both anticoagulation clinics and primary care setting in Sweden. Thromb Res 2015;136:216-20.

20. Ludvigsson JF, Andersson E, Ekbom A, et al. External review and validation of the Swedish national inpatient register. BMC Public Health 2011;11:450.

21. Köster M, Asplund K, Johansson Å, et al. Refinement of Swedish administrative registers to monitor stroke events on the national level. Neuroepidemiology 2013;40:240-6.

22. Ingelsson E, Arnlöv J, Sundström J, et al. The validity of a diagnosis of heart failure in a hospital discharge register. Eur $J$ Heart Fail 2005;7:787-91.

23. Smith JG, Platonov PG, Hedblad B, et al. Atrial fibrillation in the Malmö Diet and Cancer study: a study of occurrence, risk factors and diagnostic validity. Eur J Epidemiol 2010;25:95-102.

24. Kirchhof P, Benussi S, Kotecha D, et al. 2016 ESC guidelines for the management of atrial fibrillation developed in collaboration with EACTS. Eur Heart J 2016;37:2893-962.

25. Friberg $L$, Skeppholm M, Terént $A$. Benefit of anticoagulation unlikely in patients with atrial fibrillation and a CHA2DS2-VASc score of 1. J Am Coll Cardiol 2015;65:225-32.

26. Fine JP, Gray RJ. A proportional hazards model for the subdistribution of a competing risk. J Am Stat Assoc 1999;94:496-509.

27. Graham DJ, Reichman ME, Wernecke M, et al. Cardiovascular, bleeding, and mortality risks in elderly medicare patients treated with dabigatran or warfarin for nonvalvular atrial fibrillation. Circulation 2015;131:157-64.

28. Staerk L, Fosbøl EL, Lip GYH, et al. Ischaemic and haemorrhagic stroke associated with non-vitamin $\mathrm{K}$ antagonist oral anticoagulants and warfarin use in patients with atrial fibrillation: a nationwide cohort study. Eur Heart J 2017;38:ehw496.

29. Siegbahn A, Oldgren J, Andersson U, et al. D-dimer and factor VIla in atrial fibrillation - prognostic values for cardiovascular events and effects of anticoagulation therapy. A RE-LY substudy. Thromb Haemost 2016;115:921-30. 